\title{
MULTIPLE SOLUTIONS FOR ELLIPTIC EQUATIONS INVOLVING A GENERAL OPERATOR IN DIVERGENCE FORM
}

\author{
Giovanni Molica Bisci and Dušan Repovš
}

\author{
University of Reggio Calabria, Department PAU \\ Via Melissari, 24 - 89124 Reggio Calabria, Italy; gmolica@unirc.it \\ University of Ljubljana, Faculty of Education, and Faculty of Mathematics and Physics \\ POB 2964, Ljubljana, Slovenia 1001; dusan.repovs@guest.arnes.si
}

\begin{abstract}
In this paper, exploiting variational methods, the existence of three weak solutions for a class of elliptic equations involving a general operator in divergence form and with Dirichlet boundary condition is investigated. Several special cases are analyzed. In conclusion, for completeness, a concrete example of an application is presented by finding the existence of three nontrivial weak solutions for an uniformly elliptic second-order problem on a bounded Euclidean domain.
\end{abstract}

\section{Introduction}

In this paper we study the existence of multiple weak solutions for the following Dirichlet boundary-value problem:

$$
\left(D_{\lambda}^{f}\right) \quad \begin{cases}-\operatorname{div}(a(x, \nabla u))=\lambda k(x) f(u) & \text { in } \Omega, \\ u=0 & \text { on } \partial \Omega,\end{cases}
$$

where $\Omega$ is a bounded domain in $\mathbf{R}^{N}(N \geq 2)$ with smooth boundary $\partial \Omega, p>1$, $a: \bar{\Omega} \times \mathbf{R}^{N} \rightarrow \mathbf{R}^{N}$ is a suitable continuous map of gradient type, and $\lambda$ is a positive real parameter. Further, $f: \mathbf{R} \rightarrow \mathbf{R}$ and $k: \bar{\Omega} \rightarrow \mathbf{R}^{+}$are two continuous functions.

We first observe that our multiplicity theorems are related to some known results in the current literature on the subject. For instance, we refer the reader to the paper of Brézis and Oswald [4] in which an existence and uniqueness result was obtained via the minimization technique and by using a maximum principle, as well as to the work [12], in which Lin proved the existence, uniqueness and asymptotical properties of the solutions of problem $\left(D_{\lambda}^{f}\right)$, when $f$ behaves like the power $t^{q}$, for some $\left.q \in\right] 0,1[$, and sufficiently large $t$.

Further, Dirichlet problems involving a general operator in divergence form was studied by De Nápoli and Mariani in [7]. In this paper the existence of one weak solution were proved by exploiting the standard mountain pass geometry and requiring, among other assumptions, that the nonlinearity $f$ has a $(p-1)$-superlinear behaviour at infinity. The non-uniform case was successively considered by Duc and $\mathrm{Vu}$ in [8], by extending the result of [7] under the key hypothesis that the map $a$ fulfills the growth condition:

$$
|a(x, \xi)| \leq c_{0}\left(h_{0}(x)+h_{1}(x)|\xi|^{p-1}\right),
$$

doi:10.5186/aasfm.2014.3909

2010 Mathematics Subject Classification: Primary 35J15, 35J25, 35J62, 35J92.

Key words: Three weak solutions, variational methods, divergence type equations. 
for every $(x, \xi) \in \Omega \times \mathbf{R}^{N}$, where $h_{0} \in L^{p /(p-1)}(\Omega), h_{1} \in L_{l o c}^{1}(\Omega)$ and $c_{0}$ is a positive constant.

In both papers [7] and [8] the function $f$ satisfies the celebrated AmbrosettiRabinowitz condition:

(AR) There exist $s_{0}>0$ and $\theta>p$ such that

$$
0<\theta \int_{0}^{s} f(t) d t \leq s f(s), \quad \forall|s| \geq s_{0}
$$

In [5], Colasuonno, Pucci and Varga studied different and very general classes of elliptic operators in divergence form looking at the existence of multiple weak solutions; see also Remark 3.13. Their contributions represent a nice improvement, in several directions, of the results obtained by Kristály, Lisei and Varga in [10] in which a uniform Dirichlet problem with parameter is investigated.

Yang, Geng and Yan, in [18], proved the existence of three weak solutions for singular $p$-Laplacian type equations. Further, Papageorgiou, Rocha and Staicu considered a nonsmooth $p$-Laplacian problem in divergence form, obtaining the existence of at least two nontrivial weak solutions (see [14]).

We also note that Autuori, Pucci and Varga, in [1], studied a quasilinear elliptic eigenvalue problem, for every value of the parameter $\lambda$, by using suitable variational techniques (see [15] for related topics).

A similar approach is adopted by Servadei in [16]. More precisely, in this paper, the author consider the following variational inequality involving a self-adjoint, second-order, uniformly elliptic operator $A$ without lower-order terms:

Find $u \in K$ such that

$$
\langle A v, v-u\rangle-\lambda \int_{\Omega} u(x)(v-u)(x) d x \geq \int_{\Omega} p(x) f(u(x))(v-u)(x) d x
$$

for all $v \in K$,

where $\lambda$ is a positive parameter, $p \in L^{\infty}(\Omega)$ vanishes only on a set of measure zero and positive somewhere on $\Omega, f$ is a continuous function satisfying certain superlinear and subcritical growth conditions at zero and at infinity, and $K$ is a suitable subset of $H_{0}^{1}(\Omega)$.

If $A$ is the Laplacian and if $f$ has a special form, then this problem is well understood, and the behavior of the solution depends on the value of $\lambda$ compared to the first eigenvalue of the Laplacian. In the cited paper, the author extends these results to a general setting. In particular, if $\lambda_{1}$ denotes the first eigenvalue of $A$, then there is a nontrivial nonnegative solution if $\lambda<\lambda_{1}$. The method of proof is based on the Mountain Pass Lemma. Further, the Linking Theorem was applied in the case $\lambda \geq \lambda_{1}$ (see [13] for related applications).

Motivated by this large interest in elliptic equations with operators in divergence form, the aim of this paper is to establish, by using variational arguments (see Theorem 2.1), a precise interval of values of the parameter $\lambda$ for which problem $\left(D_{\lambda}^{f}\right)$ admits at least three weak solutions, without explicit perturbations of the nonlinear term, even in the higher dimensional setting (see Theorem 3.5).

A direct consequence of Theorem 3.5 is presented in Corollary 3.9. More precisely, in this case the existence of at least two weak nontrivial solutions for problem $\left(D_{\lambda}^{f}\right)$ 
is obtained for sufficiently large $\lambda$. A special case of Corollary 3.9 is Corollary 3.10, where the classical autonomous setting is stated (see Example 3.11).

The plan of the paper is as follows. Section 2 is devoted to our abstract framework, while Section 3 is dedicated to the main results. A concrete example of an application is then presented (see Example 3.12). Finally, we cite a recent monograph by Kristály, Rădulescu and Varga [11] as a general reference on variational methods adopted here.

\section{Abstract framework}

Let $\Omega$ be a bounded domain in $\mathbf{R}^{N}$ with smooth boundary $\partial \Omega, p>1$, and denote by:

○ $X$ the Sobolev space $W_{0}^{1, p}(\Omega)$ endowed by the norm

$$
\|u\|:=\left(\int_{\Omega}|\nabla u(x)|^{p} d x\right)^{1 / p}
$$

○ $X^{*}$ the topological dual of $X$;

$\circ\langle\cdot, \cdot\rangle$ the duality brackets for the pair $\left(X^{*}, X\right)$.

In addition, as customary, the symbol

$$
p^{*}:= \begin{cases}p N /(N-p) & \text { if } \quad 1<p<N \\ \infty & \text { if } \quad p \geq N\end{cases}
$$

denotes the critical Sobolev exponent of $p$. Fixing $q \in\left[1, p^{*}\right]$, by the Sobolev embedding theorem, there exists a positive constant $c_{q}$ such that

$$
\|u\|_{L^{q}(\Omega)} \leq c_{q}\|u\|, \quad u \in X,
$$

and, in particular, the embedding $X \hookrightarrow L^{q}(\Omega)$ is compact for every $q \in\left[1, p^{*}[\right.$. Moreover, if $1<p<N$, the best constant $c_{p^{*}}$ is given by

$$
c_{p^{*}}=\frac{1}{N \sqrt{\pi}}\left(\frac{N ! \Gamma\left(\frac{N}{2}\right)}{2 \Gamma\left(\frac{N}{p}\right) \Gamma\left(N+1-\frac{N}{p}\right)}\right)^{1 / N} \eta^{1-1 / p},
$$

where

$$
\eta:=\frac{N(p-1)}{N-p}
$$

see, for instance, [17]. If $p>N$, let

$$
\sup \left\{\frac{\max _{x \in \bar{\Omega}}|u(x)|}{\|u\|}: u \in W_{0}^{1, p}(\Omega), u \neq 0\right\}<+\infty .
$$

It is well-known $[17$, formula $(6 \mathrm{~b})]$ that by putting

$$
m:=\frac{N^{-\frac{1}{p}}}{\sqrt{\pi}}\left[\Gamma\left(1+\frac{N}{2}\right)\right]^{\frac{1}{N}}\left(\frac{p-1}{p-N}\right)^{1-\frac{1}{p}}(\operatorname{meas}(\Omega))^{\frac{1}{N}-\frac{1}{p}},
$$

one has

$$
\|u\|_{\infty}:=\max _{x \in \bar{\Omega}}|u(x)| \leq m\|u\|
$$


for every $u \in X$ (equality occurs when $\Omega$ is a ball). Here $\Gamma$ is the Gamma function defined by

$$
\Gamma(t):=\int_{0}^{+\infty} z^{t-1} e^{-z} d z, \quad \forall t>0
$$

and "meas $(\Omega)$ " denotes the usual Lebesgue measure of $\Omega$. Moreover, let

$$
\tau:=\sup _{x \in \Omega} \operatorname{dist}(x, \partial \Omega) .
$$

Simple calculations show that there is $x_{0} \in \Omega$ such that $B\left(x_{0}, \tau\right) \subseteq \Omega$, where $B\left(x_{0}, \tau\right)$ is the open ball of radius $\tau$ centered at the point $x_{0}$. We also denote by

$$
\omega_{s}:=s^{N} \frac{\pi^{N / 2}}{\Gamma\left(1+\frac{N}{2}\right)},
$$

the measure of the $N$-dimensional ball of radius $s>0$. At this point, for $\delta>0$, let $u_{\delta} \in X$ be the following function

$$
u_{\delta}(x):= \begin{cases}0 & \text { if } x \in \Omega \backslash B\left(x_{0}, \tau\right), \\ \frac{2 \delta}{\tau}\left(\tau-\left|x-x_{0}\right|\right) & \text { if } x \in B\left(x_{0}, \tau\right) \backslash B\left(x_{0}, \tau / 2\right), \\ \delta & \text { if } x \in B\left(x_{0}, \tau / 2\right),\end{cases}
$$

that will be useful in the sequel in the proof of our theorems. One has that

$$
\left\|u_{\delta}\right\|^{p}=\int_{\Omega}\left|\nabla u_{\delta}(x)\right|^{p} d x=\frac{2^{p} \delta^{p} \omega_{\tau}}{\tau^{p}}\left(1-\frac{1}{2^{N}}\right) .
$$

Indeed,

$$
\begin{aligned}
\int_{\Omega}\left|\nabla u_{\delta}(x)\right|^{p} d x & =\delta^{p} \int_{B\left(x_{0}, \tau\right) \backslash B\left(x_{0}, \tau / 2\right)} \frac{2^{p}}{\tau^{p}} d x \\
& =\frac{2^{p} \delta^{p}}{\tau^{p}}\left(\operatorname{meas}\left(B\left(x_{0}, \tau\right)\right)-\operatorname{meas}\left(B\left(x_{0}, \tau / 2\right)\right)\right) \\
& =\frac{2^{p} \delta^{p} \omega_{\tau}}{\tau^{p}}\left(1-\frac{1}{2^{N}}\right)
\end{aligned}
$$

where, from now on, "meas $\left(B\left(x_{0}, s\right)\right)$ " for $s>0$ stands for the Lebesgue measure of the open ball $B\left(x_{0}, s\right)$.

As pointed out before, our approach is variational. More precisely, the main tool will be the following abstract critical point theorem for smooth functions that can be derived from [2, Theorem 3.6].

Theorem 2.1. Let $X$ be a reflexive real Banach space and let $\Phi, \Psi: X \rightarrow \boldsymbol{R}$ be two $C^{1}$-functionals with $\Phi\left(0_{X}\right)=\Psi\left(0_{X}\right)=0$ and such that:

$\left(\mathrm{a}_{1}\right) \Phi$ is coercive and sequentially weakly lower semicontinuous;

$\left(\mathrm{a}_{2}\right) \Psi: X \rightarrow \boldsymbol{R}$ is sequentially weakly upper semicontinuous.

For every $\lambda>0$, put $J_{\lambda}:=\Phi-\lambda \Psi$ and assume that the following conditions are satisfied:

$\left(\mathrm{a}_{3}\right)$ There exist $r>0$ and $\bar{u} \in X$, with $r<\Phi(\bar{u})$, such that

$$
\frac{\sup _{\left.\left.u \in \Phi^{-1}(]-\infty, r\right]\right)} \Psi(u)}{r}<\frac{\Psi(\bar{u})}{\Phi(\bar{u})}
$$


$\left(\mathrm{a}_{4}\right)$ For each $\left.\lambda \in \Lambda_{r}:=\right] \frac{\Phi(\bar{u})}{\Psi(\bar{u})}, \frac{r}{\sup _{\left.\left.u \in \Phi^{-1}(]-\infty, r\right]\right)} \Psi(u)}\left[\right.$ the functional $J_{\lambda}$ is bounded from below and fulfills (PS) ${ }_{\mu}$, with $\mu \in \mathbf{R}$.

Then for each $\lambda \in \Lambda_{r}$, the functional $J_{\lambda}$ has at least three distinct critical points in $X$.

For the sake of completeness, we also recall that the $C^{1}$-functional $J_{\lambda}: X \rightarrow \mathbf{R}$ satisfies the Palais-Smale condition at level $\mu \in \mathbf{R}$ when:

$(\mathrm{PS})_{\mu}$ Every sequence $\left\{u_{n}\right\} \subset X$ such that

$$
J_{\lambda}\left(u_{n}\right) \rightarrow \mu \text { and }\left\|J_{\lambda}^{\prime}\left(u_{n}\right)\right\|_{X^{*}} \rightarrow 0
$$

as $n \rightarrow \infty$, possesses a convergent subsequence in $X$.

\section{Main results}

In the sequel, let $p>1$ and let $\Omega \subset \mathbf{R}^{N}$ be a bounded Euclidean domain, where $N \geq 2$. Further, let $A: \bar{\Omega} \times \mathbf{R}^{N} \rightarrow \mathbf{R}$, and let $A=A(x, \xi)$ be a continuous function in $\bar{\Omega} \times \mathbf{R}^{N}$, with continuous gradient $a(x, \xi):=\nabla_{\xi} A(x, \xi): \bar{\Omega} \times \mathbf{R}^{N} \rightarrow \mathbf{R}^{N}$, and assume that the following conditions hold:

$\left(\alpha_{1}\right) A(x, 0)=0$ for all $x \in \Omega$;

$\left(\alpha_{2}\right)$ a satisfies the growth condition $|a(x, \xi)| \leq c\left(1+|\xi|^{p-1}\right)$ for all $x \in \Omega, \xi \in \boldsymbol{R}^{N}$, $c>0$

$\left(\alpha_{3}\right) A$ is p-uniformly convex, that is

$$
A\left(x, \frac{\xi+\eta}{2}\right) \leq \frac{1}{2} A(x, \xi)+\frac{1}{2} A(x, \eta)-k|\xi-\eta|^{p},
$$

for every $x \in \bar{\Omega}, \xi, \eta \in \boldsymbol{R}^{N}$ and some $k>0$;

$\left(\alpha_{4}\right) A$ is $p$-subhomogeneous, i.e., $0 \leq a(x, \xi) \cdot \xi \leq p A(x, \xi)$ for all $x \in \bar{\Omega}, \xi \in \boldsymbol{R}^{N}$;

$\left(\alpha_{5}\right) A$ satisfies $\Lambda_{1}|\xi|^{p} \leq A(x, \xi) \leq \Lambda_{2}|\xi|^{p}$ for all $x \in \bar{\Omega}, \xi \in \boldsymbol{R}^{N}$, where $\Lambda_{1}$ and $\Lambda_{2}$ are positive constants.

Example 3.1. Note that by choosing

$$
A(x, \xi):=\frac{|\xi|^{p}}{p}
$$

with $p \geq 2$, we have the usual $p$-Laplacian operator. See also Remark 3.7 for details on this special case.

Remark 3.2. Define $A^{|\vee|}: \bar{\Omega} \times \mathbf{R} \rightarrow \mathbf{R}$ as follows,

$$
A^{|\mathrm{v}|}(x, t):=\sup _{|\xi|=t} A(x, \xi), \quad \forall x \in \bar{\Omega} .
$$

For every $\varepsilon, b \in(0,1)$ and $x \in \bar{\Omega}$, let us put $E_{\varepsilon, b}(x)$ to be the set

$$
\begin{array}{r}
\left\{(\xi, \eta) \in \mathbf{R}^{N} \times \mathbf{R}^{N}: A\left(x, \frac{\xi-\eta}{2}\right) \geq \frac{1}{2} \max \{A(x, \varepsilon \xi), A(x, \varepsilon \eta)\},\right. \\
\left.A\left(x, \frac{\xi+\eta}{2}\right)>(1-b) \frac{A(x, \xi)+A(x, \eta)}{2}\right\},
\end{array}
$$


and

$$
q_{\varepsilon, b}(x):=\sup \left\{\frac{|\xi-\eta|}{2}:(\xi, \eta) \in E_{\varepsilon, b}(x)\right\} .
$$

It is known that the map $A$ is said to be uniformly convex if it satisfies

$$
\lim _{b \rightarrow 0} \int_{\Omega} A^{|\mathrm{\vee}|}\left(x, q_{\varepsilon, b}(x)\right) d x=0, \quad \text { for every } \varepsilon \in(0,1) .
$$

As pointed out in [9, Remark 2.2], we just observe that our hypotheses $\left(\alpha_{1}\right)-\left(\alpha_{5}\right)$ imply that $A$ is an uniformly convex operator.

Remark 3.3. Integrating $\left(\alpha_{2}\right)$ we deduce that the map $A$ satisfies the growth condition:

$$
A(x, \xi) \leq c\left(|\xi|+|\xi|^{p}\right),
$$

for every $x \in \bar{\Omega}$ and $\xi \in \mathbf{R}^{N}$. Indeed, we have

$$
A(x, \xi)=\int_{0}^{1} \frac{d}{d t} A(x, t \xi) d t=\int_{0}^{1} a(x, t \xi) \cdot \xi d t .
$$

Hence, we deduce that

$$
A(x, \xi) \leq c \int_{0}^{1}\left(1+|\xi|^{p-1} t^{p-1}\right)|\xi| d t \leq c|\xi|+\frac{c}{p}|\xi|^{p} \leq c\left(|\xi|+|\xi|^{p}\right),
$$

for every $x \in \bar{\Omega}$ and $\xi \in \mathbf{R}^{N}$. Moreover, we also note that by condition $\left(\alpha_{3}\right)$, the functional $\Phi: X \rightarrow \mathbf{R}$ defined by

$$
u \mapsto \int_{\Omega} A(x, \nabla u(x)) d x
$$

is a locally uniformly convex operator. Taking into account the above facts, its Gâteaux derivative $\Phi^{\prime}: X \rightarrow X^{*}$ satisfies the $\left(S_{+}\right)$condition; see [7, Proposition 2.1]:

$\left(S_{+}\right)$For every sequence $\left\{u_{n}\right\} \subset X$ such that $u_{n} \rightarrow u$ (weakly) in $X$ and

$$
\limsup _{n \rightarrow \infty} \int_{\Omega} a\left(x, \nabla u_{n}(x)\right) \cdot \nabla\left(u_{n}-u\right)(x) d x \leq 0,
$$

it follows that $u_{n} \rightarrow u$ (strongly) in $X$. $\mathfrak{F}_{q}$ if

From now on, we say that a continuous function $f: \mathbf{R} \rightarrow \mathbf{R}$ belongs to the class

$$
|f(t)| \leq a_{1}+a_{2}|t|^{q-1}, \quad(\forall t \in \mathbf{R})
$$

for some nonnegative constants $a_{1}, a_{2}$, where $\left.q \in\right] 1, p N /(N-p)[$ if $p<N$ and $1<q<+\infty$ if $p \geq N$.

Let $\Psi: X \rightarrow \mathbf{R}$ be defined

$$
\Psi(u):=\int_{\Omega} k(x) F(u(x)) d x,
$$

where $k: \bar{\Omega} \rightarrow \mathbf{R}$ is a positive and continuous function, and

$$
F(s):=\int_{0}^{s} f(t) d t
$$

for every $s \in \mathbf{R}$. 
Fixing $\lambda>0$, we say that $u \in X$ is a weak solution of problem $\left(D_{\lambda}^{f}\right)$ if

$$
\int_{\Omega} a(x, \nabla u(x)) \cdot \nabla v(x) d x=\lambda \int_{\Omega} k(x) f(u(x)) v(x) d x,
$$

for every $v \in X$.

In the sequel we look at the existence of weak solutions of problem $\left(D_{\lambda}^{f}\right)$ finding critical points for a suitable associated energy functional $J_{\lambda}$.

Lemma 3.4. Let us assume that $f \in \mathfrak{F}_{q}$. Then the functionals $\Phi$ and $\Psi$ are respectively sequentially weakly lower and upper semicontinuous. Hence in particular, for every $\lambda \in \boldsymbol{R}^{+}$, the functional $J_{\lambda}: X \rightarrow \boldsymbol{R}$ given by

$$
J_{\lambda}:=\Phi-\lambda \Psi
$$

is sequentially weakly lower semicontinuous.

Proof. The functional $\Phi$ being locally uniformly convex, is weakly lower semicontionous. On the other hand, since $f \in \mathfrak{F}_{q}$ and $k \in C^{0}(\bar{\Omega})$, we have $|k(x) f(t)| \leq$ $\|k\|_{\infty}\left(a_{1}+a_{2}|t|^{q-1}\right)$, for every $(x, t) \in \bar{\Omega} \times \mathbf{R}$. Finally, due to the fact that the embedding $X \hookrightarrow L^{q}(\Omega)$ is compact, we obtain that $\Psi$ is sequentially weakly (upper) semicontinuous in the standard way.

Set

$$
\kappa:=\left(\frac{2^{N-p}}{\omega_{\tau}\left(2^{N}-1\right) \Lambda_{1}}\right)^{1 / p} \tau
$$

and

$$
G_{1}:=\left(\frac{2^{p}\left(2^{N}-1\right) \Lambda_{2}}{\tau^{p} \min _{x \in \bar{\Omega}} k(x)}\right) \frac{\|k\|_{\infty} c_{1}}{\Lambda_{1}^{1 / p}},
$$

as well as

$$
G_{2}:=\left(\frac{2^{p}\left(2^{N}-1\right) \Lambda_{2}}{\tau^{p} \min _{x \in \bar{\Omega}} k(x)}\right) \frac{\|k\|_{\infty} c_{q}^{q}}{q \Lambda_{1}^{q / p}} .
$$

Our main result is as follows.

Theorem 3.5. Let $f \in \mathfrak{F}_{q}$ and assume that

$\left(\mathrm{h}_{0}\right) F(s) \geq 0$ for every $s \in \boldsymbol{R}^{+}$,

$\left(\mathrm{h}_{1}\right) \lim _{|t| \rightarrow \infty} \frac{f(t)}{|t|^{p-1}}=0$,

$\left(\mathrm{h}_{2}\right)$ there are positive constants $\gamma$ and $\delta$, with $\delta>\gamma \kappa$, such that

$$
\frac{F(\delta)}{\delta^{p}}>a_{1} \frac{G_{1}}{\gamma^{p-1}}+a_{2} G_{2} \gamma^{q-p} .
$$

Then for each parameter $\lambda$ belonging to

$$
\left.\Lambda_{(\gamma, \delta)}:=\frac{2^{p}\left(2^{N}-1\right) \Lambda_{2}}{\tau^{p} \min _{x \in \bar{\Omega}} k(x)}\right] \frac{\delta^{p}}{F(\delta)}, \frac{1}{\left(a_{1} \frac{G_{1}}{\gamma^{p-1}}+a_{2} G_{2} \gamma^{q-p}\right)}[,
$$

problem $\left(D_{\lambda}^{f}\right)$ possesses at least three weak solutions. 
Proof. Our aim is to apply Theorem 2.1. Let $X:=W_{0}^{1, p}(\Omega)$ and consider the functionals $\Phi, \Psi: X \rightarrow \mathbf{R}$ defined before. Clearly, $\Phi: X \rightarrow \mathbf{R}$ is coercive since, by condition $\left(\alpha_{5}\right)$, it follows that

$$
\Phi(u) \geq \Lambda_{1}\|u\|^{p} \rightarrow+\infty,
$$

when $\|u\| \rightarrow \infty$. Moreover, $\Phi$ is a continuously Gâteaux differentiable and sequentially weakly lower semicontinuous functional (by Lemma 3.4). On the other hand, $\Psi$ is well-defined, continuously Gâteaux differentiable and, again by Lemma 3.4, a weakly upper semicontinuous functional. More precisely, one has

$$
\begin{aligned}
& \Phi^{\prime}(u)(v)=\int_{\Omega} a(x, \nabla u(x)) \cdot \nabla v(x) d x, \\
& \Psi^{\prime}(u)(v)=\int_{\Omega} k(x) f(u(x)) v(x) d x,
\end{aligned}
$$

for every $u, v \in X$. Hence conditions $\left(\mathrm{a}_{1}\right)$ and $\left(\mathrm{a}_{2}\right)$ of Theorem 2.1 are satisfied. Now, fix $\lambda>0$. A critical point of the functional $J_{\lambda}:=\Phi-\lambda \Psi$ is a function $u \in X$ such that

$$
\Phi^{\prime}(u)(v)-\lambda \Psi^{\prime}(u)(v)=0,
$$

for every $v \in X$. Hence, the critical points of the functional $J_{\lambda}$ are weak solutions of problem $\left(D_{\lambda}^{f}\right)$. Moreover, $\Phi\left(0_{X}\right)=\Psi\left(0_{X}\right)=0$. Since $f \in \mathfrak{F}_{q}$, one has that

$$
F(s) \leq a_{1}|s|+a_{2} \frac{|s|^{q}}{q}
$$

for every $s \in \mathbf{R}$.

Let $r \in] 0,+\infty[$ and consider the function

$$
\chi(r):=\frac{\sup _{\left.\left.u \in \Phi^{-1}(]-\infty, r\right]\right)} \Psi(u)}{r} .
$$

Taking into account (6), it follows that

$$
\Psi(u)=\int_{\Omega} k(x) F(u(x)) d x \leq\|k\|_{\infty}\left(a_{1}\|u\|_{L^{1}(\Omega)}+\frac{a_{2}}{q}\|u\|_{L^{q}(\Omega)}^{q}\right) .
$$

Then let $u \in X$ be such that $\Phi(u) \leq r$, that is

$$
\int_{\Omega} A(x, \nabla u(x)) d x \leq r .
$$

Hence the above relation together with condition $\left(\alpha_{5}\right)$ implies that

$$
\|u\| \leq \frac{r^{1 / p}}{\Lambda_{1}^{1 / p}}
$$

In other words, one has the following algebraic inclusion

$$
\left\{u \in X: \int_{\Omega} A(x, \nabla u(x)) d x \leq r\right\} \subseteq\left\{u \in X:\|u\| \leq \frac{r^{1 / p}}{\Lambda_{1}^{1 / p}}\right\} .
$$

Consequently, by the Sobolev inequalities (1), one has

$$
\Psi(u) \leq\|k\|_{\infty}\left(\frac{c_{1} a_{1} r^{1 / p}}{\Lambda_{1}^{1 / p}}+\frac{c_{q}^{q} a_{2} r^{q / p}}{q \Lambda_{1}^{q / p}}\right)
$$


for every $\left.\left.u \in \Phi^{-1}(]-\infty, r\right]\right)$. Hence,

$$
\sup _{\left.\left.u \in \Phi^{-1}(]-\infty, r\right]\right)} \Psi(u) \leq\|k\|_{\infty}\left(\frac{c_{1} a_{1} r^{1 / p}}{\Lambda_{1}^{1 / p}}+\frac{c_{q}^{q} a_{2} r^{q / p}}{q \Lambda_{1}^{q / p}}\right) .
$$

By the above inequality, the following relation holds

$$
\chi(r) \leq\|k\|_{\infty} L(r),
$$

for every $r>0$, where we set

$$
L(r):=\frac{c_{1} a_{1} r^{1 / p-1}}{\Lambda_{1}^{1 / p}}+\frac{c_{q}^{q} a_{2} r^{q / p-1}}{q \Lambda_{1}^{q / p}} .
$$

Next, let $u_{\delta}$ be the function defined in Section 2. Clearly $u_{\delta} \in X$ and, since $\left(\alpha_{5}\right)$ holds, we have

$$
\Phi\left(u_{\delta}\right)=\int_{\Omega} A\left(x, \nabla u_{\delta}(x)\right) d x \leq \Lambda_{2}\left(\frac{2^{p} \delta^{p} \omega_{\tau}}{\tau^{p}}\left(1-\frac{1}{2^{N}}\right)\right) .
$$

Taking into account that $\delta>\gamma \kappa$, by a direct computation, one has $\gamma^{p}<\Phi\left(u_{\delta}\right)$. Moreover from $\left(\mathrm{h}_{0}\right)$ and taking into account that the map $k$ is positive and continuous in $\bar{\Omega}$, we easily derive

$$
\int_{\Omega} k(x) F\left(u_{\delta}(x)\right) d x \geq F(\delta) \frac{\pi^{N / 2}}{\Gamma(1+N / 2)} \frac{\tau^{N}}{2^{N}} \min _{x \in \bar{\Omega}} k(x) .
$$

Hence, by (8) and (9), one has

$$
\frac{\Psi\left(u_{\delta}\right)}{\Phi\left(u_{\delta}\right)} \geq\left(\frac{\tau^{p} \min _{x \in \bar{\Omega}} k(x)}{2^{p}\left(2^{N}-1\right) \Lambda_{2}}\right) \frac{F(\delta)}{\delta^{p}} .
$$

In view of $\left(\mathrm{h}_{2}\right)$ and taking into account $(7)$ and $(10)$, we get

$$
\begin{aligned}
\chi\left(\gamma^{p}\right) & =\frac{\sup _{\left.\left.u \in \Phi^{-1}(]-\infty, \gamma^{p}\right]\right)} \Psi(u)}{\gamma^{p}} \leq\|k\|_{\infty} L\left(\gamma^{p}\right)=\frac{\tau^{p} \min _{x \in \bar{\Omega}} k(x)}{2^{p}\left(2^{N}-1\right) \Lambda_{2}}\left(a_{1} \frac{G_{1}}{\gamma^{p-1}}+a_{2} G_{2} \gamma^{q-p}\right) \\
& <\left(\frac{\tau^{p} \min _{x \in \bar{\Omega}} k(x)}{2^{p}\left(2^{N}-1\right) \Lambda_{2}}\right) \frac{F(\delta)}{\delta^{p}} \leq \frac{\Psi\left(u_{\delta}\right)}{\Phi\left(u_{\delta}\right)} .
\end{aligned}
$$

Therefore, assumption $\left(\mathrm{a}_{3}\right)$ of Theorem 2.1 is satisfied.

Let us fix $\lambda>0$. By condition $\left(\mathrm{h}_{1}\right)$, there exists $\delta_{\lambda}$ such that

$$
|f(t)| \leq \frac{\Lambda_{1}}{c_{p}^{p}(1+\lambda)\|k\|_{\infty}}|t|^{p-1}
$$

for every $|t| \geq \delta_{\lambda}$. By integration we get

for every $s \in \mathbf{R}$. Thus

$$
|F(s)| \leq \frac{\Lambda_{1}}{c_{p}^{p}(1+\lambda)\|k\|_{\infty}}|s|^{p}+\max _{|t| \leq \delta_{\lambda}}|f(t) \| s|,
$$

$$
\begin{aligned}
J_{\lambda}(u) & \geq \Phi(u)-\lambda|\Psi(u)| \\
& \geq \Lambda_{1}\|u\|^{p}-\frac{\Lambda_{1} \lambda}{(1+\lambda)}\|u\|^{p}-c_{1}\|k\|_{\infty} \lambda \max _{|t| \leq \delta_{\lambda}}|f(t)|\|u\| .
\end{aligned}
$$


Then the functional $J_{\lambda}$ is bounded from below and, since $p>1, J_{\lambda}(u) \rightarrow+\infty$ whenever $\|u\| \rightarrow+\infty$. Hence $J_{\lambda}$ is coercive. Now, let us prove that $J_{\lambda}$ satisfies the condition (PS) ${ }_{\mu}$ for $\mu \in \mathbf{R}$. For our goal, let $\left\{u_{n}\right\} \subset X$ be a Palais-Smale sequence, i.e.

$$
J_{\lambda}\left(u_{n}\right) \rightarrow \mu, \quad \text { and } \quad\left\|J_{\lambda}^{\prime}\left(u_{n}\right)\right\|_{X^{*}} \rightarrow 0 .
$$

Taking into account the coercivity of $J_{\lambda}$, the sequence $\left\{u_{n}\right\}$ is necessarily bounded in $X$. Since $X$ is reflexive, we may extract a subsequence, which for simplicity we call again $\left\{u_{n}\right\}$, such that $u_{n} \rightarrow u$ in $X$. We will prove that $\left\{u_{n}\right\}$ strongly converges to $u \in X$. Exploiting the derivative $J_{\lambda}^{\prime}\left(u_{n}\right)\left(u_{n}-u\right)$, we obtain

$$
\begin{aligned}
& \int_{\Omega} a\left(x, \nabla u_{n}(x)\right) \cdot \nabla\left(u_{n}-u\right)(x) d x \\
& =\left\langle J_{\lambda}^{\prime}\left(u_{n}\right), u_{n}-u\right\rangle+\lambda \int_{\Omega} k(x) f\left(u_{n}(x)\right)\left(u_{n}-u\right)(x) d x .
\end{aligned}
$$

Since $\left\|J_{\lambda}^{\prime}\left(u_{n}\right)\right\|_{X^{*}} \rightarrow 0$ and the sequence $\left\{u_{n}-u\right\}$ is bounded in $X$, taking into account that $\left|\left\langle J_{\lambda}^{\prime}\left(u_{n}\right), u_{n}-u\right\rangle\right| \leq\left\|J_{\lambda}^{\prime}\left(u_{n}\right)\right\|_{X^{*}}\left\|u_{n}-u\right\|$, one has

$$
\left\langle J_{\lambda}^{\prime}\left(u_{n}\right), u_{n}-u\right\rangle \rightarrow 0 \text {. }
$$

Further, by the asymptotic condition $\left(\mathrm{h}_{1}\right)$, there exists a real positive constant $c$ such that $|f(t)| \leq c\left(1+|t|^{p-1}\right)$, for every $t \in \mathbf{R}$. Then

$$
\begin{aligned}
& \int_{\Omega} k(x)\left|f\left(u_{n}(x)\right)\right|\left|u_{n}(x)-u(x)\right| d x \\
& \leq \sigma \int_{\Omega}\left|u_{n}(x)-u(x)\right| d x+\sigma \int_{\Omega}\left|u_{n}(x)\right|^{p-1}\left|u_{n}(x)-u(x)\right| d x \\
& \leq \sigma\left((\operatorname{meas}(\Omega))^{1 / p^{\prime}}+\left\|u_{n}\right\|_{L^{p}(\Omega)}^{p-1}\right)\left\|u_{n}-u\right\|_{L^{p}(\Omega)},
\end{aligned}
$$

where $\sigma:=c\|k\|_{\infty}$ and $p^{\prime}$ is the conjugate exponent of $p$.

Now, the embedding $W^{1, p}(\Omega) \hookrightarrow L^{p}(\Omega)$ is compact, hence $u_{n} \rightarrow u$ strongly in $L^{p}(\Omega)$. So we obtain

$$
\int_{\Omega} k(x)\left|f\left(u_{n}(x)\right)\right|\left|u_{n}(x)-u(x)\right| d x \rightarrow 0 .
$$

We may conclude

$$
\limsup _{n \rightarrow \infty}\left\langle a\left(x, u_{n}\right), u_{n}-u\right\rangle=0
$$

where $\left\langle a\left(x, u_{n}\right), u_{n}-u\right\rangle$ denotes

$$
\int_{\Omega} a\left(x, \nabla u_{n}(x)\right) \cdot \nabla\left(u_{n}-u\right)(x) d x .
$$

But as observed in Remark 3.3, the operator $\Phi^{\prime}$ has the $\left(S_{+}\right)$property. So, in conclusion, $u_{n} \rightarrow u$ strongly in $X$. Hence, $J_{\lambda}$ is bounded from below and fulfills $(\mathrm{PS})_{\mu}$ (with $\mu \in \mathbf{R}$ ), for every positive parameter, in particular, for every

$$
\left.\lambda \in \Lambda_{(\gamma, \delta)} \subseteq\right] \frac{\Phi\left(u_{\delta}\right)}{\Psi\left(u_{\delta}\right)}, \frac{\gamma^{p}}{\sup _{\left.\left.u \in \Phi^{-1}(]-\infty, \gamma^{p}\right]\right)} \Psi(u)}[.
$$


Then also condition $\left(\mathrm{a}_{4}\right)$ holds. Hence all the assumptions of Theorem 2.1 are satisfied. Consequently, for each $\lambda \in \Lambda_{(\gamma, \delta)}$, the functional $J_{\lambda}$ has at least three distinct critical points that are weak solutions of the problem $\left(D_{\lambda}^{f}\right)$.

Remark 3.6. A careful analysis of the proof of Theorem 3.5 shows that condition $\left(\mathrm{h}_{0}\right)$ can be replaced by a more general sign condition on the potential:

$\left(\mathrm{h}_{0}^{\prime}\right) F(s) \geq 0$, for every $\left.s \in\right] 0, \delta[$,

where $\delta$ is the constant that appears in hypothesis $\left(\mathrm{h}_{2}\right)$.

\section{Remark 3.7. If}

$$
a(x, \nabla u):=|\nabla u|^{p-2} \nabla u, \quad p \geq 2,
$$

for every $x \in \bar{\Omega}$, the geometrical constants in the main result assume simpler expressions

$$
G_{1}:=\left(\frac{2^{p}\left(2^{N}-1\right)}{\tau^{p} \min _{x \in \bar{\Omega}} k(x)}\right) \frac{\|k\|_{\infty} c_{1}}{p^{\frac{p-1}{p}}}, \quad G_{2}:=\left(\frac{2^{p}\left(2^{N}-1\right)}{\tau^{p} \min _{x \in \bar{\Omega}} k(x)}\right) \frac{\|k\|_{\infty} c_{q}^{q}}{q p^{\frac{p-q}{p}}} .
$$

In this setting, under the same hypotheses of Theorem 3.5, for each parameter $\lambda$ belonging to

$$
\left.\widetilde{\Lambda}_{(\gamma, \delta)}:=\frac{2^{p}\left(2^{N}-1\right)}{p \tau^{p} \min _{x \in \bar{\Omega}} k(x)}\right] \frac{\delta^{p}}{F(\delta)}, \frac{1}{\left(a_{1} \frac{G_{1}}{\gamma^{p-1}}+a_{2} G_{2} \gamma^{q-p}\right)}[,
$$

the following problem

$$
\begin{cases}-\Delta_{p} u=\lambda k(x) f(u) & \text { in } \Omega, \\ u=0 & \text { on } \partial \Omega,\end{cases}
$$

where $\Delta_{p}:=\operatorname{div}\left(|\nabla u|^{p-2} \nabla u\right)$ denotes the $p$-Laplacian operator, possesses at least three weak solutions. The case $p=2$ has been studied in [3, Theorem 3.1] by using a similar variational approach. See also the work [6] for the Neumann setting.

Remark 3.8. An explicit upper bound for the constants $c_{q}$ in Theorem 3.5 can be easily obtained. Indeed, let $1<p<N$ and fix $q \in\left[1, p^{*}\right.$. By formula (2) one has

$$
c_{q} \leq \frac{\operatorname{meas}(\Omega)^{\frac{p^{*}-q}{p^{*} q}}}{N \sqrt{\pi}}\left(\frac{N ! \Gamma\left(\frac{N}{2}\right)}{2 \Gamma\left(\frac{N}{p}\right) \Gamma\left(N+1-\frac{N}{p}\right)}\right)^{1 / N} \eta^{1-1 / p},
$$

where

$$
\eta:=\frac{N(p-1)}{N-p}
$$

On the other hand, if $p>N$, due to inequality (4), we also have

$$
c_{q} \leq \frac{N^{-\frac{1}{p}}}{\sqrt{\pi}}\left[\Gamma\left(1+\frac{N}{2}\right)\right]^{\frac{1}{N}}\left(\frac{p-1}{p-N}\right)^{1-\frac{1}{p}}(\operatorname{meas}(\Omega))^{\frac{1}{N}+\frac{p-q}{q p}} .
$$

We also have the following multiplicity result that can be proved by Theorem 3.5. 
Corollary 3.9. Assume that $f: \boldsymbol{R} \rightarrow \boldsymbol{R}$ is a continuous and nonnegative function such that

$$
F(s) \leq a_{2} s^{q-p}, \quad\left(\forall s \in \boldsymbol{R}^{+}\right)
$$

for some $a_{2}>0$ and $p<q<p^{*}$. In addition, suppose that condition $\left(\mathrm{h}_{1}\right)$ holds. Then for every

$$
\lambda>\frac{2^{p}\left(2^{N}-1\right) \Lambda_{2}}{\tau^{p} \min _{x \in \bar{\Omega}} k(x)} \inf _{\delta>0} \frac{\delta^{p}}{F(\delta)},
$$

problem $\left(D_{\lambda}^{f}\right)$ has at least two distinct, nontrivial weak solutions.

Proof. Since $f$ is a nonnegative function, hypothesis $\left(\mathrm{h}_{0}\right)$ clearly holds. Now, fixing $\bar{\lambda}$ as in the conclusion, there exists $\bar{\delta}>0$ such that

$$
\bar{\lambda}>\left(\frac{2^{p}\left(2^{N}-1\right) \Lambda_{2}}{\tau^{p} \min _{x \in \bar{\Omega}} k(x)}\right) \frac{\bar{\delta}^{p}}{F(\bar{\delta})} .
$$

Moreover, picking a positive constant $\bar{\gamma}$, with

$$
\bar{\gamma}<\min \left\{\frac{\bar{\delta}}{\kappa},\left(\frac{2^{p}\left(2^{N}-1\right) \Lambda_{2}}{\lambda a_{2} \tau^{p} G_{2} \min _{x \in \bar{\Omega}} k(x)}\right)^{\frac{1}{q-p}}\right\},
$$

condition $\left(\mathrm{h}_{2}\right)$ is verified and, since

$$
\left.\bar{\lambda} \in \frac{2^{p}\left(2^{N}-1\right) \Lambda_{2}}{\tau^{p} \min _{x \in \bar{\Omega}} k(x)}\right] \frac{\bar{\delta}^{p}}{F(\bar{\delta})}, \frac{1}{a_{2} G_{2} \bar{\gamma}^{q-p}}[,
$$

Theorem 3.5 ensures that problem $\left(D_{\bar{\lambda}}^{f}\right)$ admits at least two nontrivial weak solutions.

The next is a special case of the above result.

Corollary 3.10. Let $2 \leq p<N$ and assume that $f: \boldsymbol{R} \rightarrow \boldsymbol{R}$ is a continuous and nonnegative function that is $(p-1)$-sublinear at infinity, i.e.

$$
\lim _{|t| \rightarrow \infty} \frac{f(t)}{|t|^{p-1}}=0 .
$$

Suppose that

$$
F(s) \leq a_{2} s^{q-p}, \quad\left(\forall s \in \boldsymbol{R}^{+}\right)
$$

for some $a_{2}>0$ and $p<q<p N /(N-p)$. Then for every

$$
\lambda>\frac{2^{p}\left(2^{N}-1\right)}{p \tau^{p}} \inf _{\delta>0} \frac{\delta^{p}}{\int_{0}^{\delta} f(t) d t},
$$

the following Dirichlet problem

$$
\begin{cases}-\Delta_{p} u=\lambda f(u) & \text { in } \Omega, \\ u=0 & \text { on } \partial \Omega,\end{cases}
$$

has at least two distinct, nontrivial weak solutions in $W_{0}^{1, p}(\Omega)$. 
Example 3.11. Let $\Omega \subset \mathbf{R}^{5}$ be a domain with smooth boundary. Taking $p=$ 4 , an example of map that satisfies all the assumptions of Corollary 3.10 is the nonnegative function $g: \mathbf{R} \rightarrow \mathbf{R}$ given by $g(t):=\log \left(1+t^{4}\right)$. Indeed,

$$
\lim _{|t| \rightarrow \infty} \frac{\log \left(1+t^{4}\right)}{|t|^{3}}=0
$$

and its potential

$$
\begin{aligned}
G(s)= & \sqrt{2} \arctan (\sqrt{2} s-1)+\sqrt{2} \arctan (\sqrt{2} s+1) \\
& -\frac{\sqrt{2}}{2} \log \left(\frac{s^{2}-\sqrt{2} s+1}{s^{2}+\sqrt{2} s+1}\right)+s \log \left(1+s^{4}\right)-4 s,
\end{aligned}
$$

satisfies $G(s) \leq s^{2}$, for every $s \in \mathbf{R}$. Then, for every

$$
\lambda>\frac{124}{\tau^{4}} \inf _{\delta>0} \frac{\delta^{4}}{\int_{0}^{\delta} g(t) d t} \approx \frac{752}{\tau^{4}},
$$

the following Dirichlet problem

$$
\begin{cases}-\Delta_{4} u=\lambda g(u) & \text { in } \Omega \\ u=0 & \text { on } \partial \Omega\end{cases}
$$

has at least two distinct, nontrivial weak solutions in $W_{0}^{1,4}(\Omega)$.

In conclusion we present an application that is a direct consequence of Theorem 3.5.

Example 3.12. Let $\left(a^{i j}\right) \in C^{0}\left(\bar{\Omega} ; \mathbf{R}^{N \times N}\right)$ be a positive definite matrix such that:

$\left(\beta_{1}\right) a^{i j}(x)=a^{j i}(x)$, for every $x \in \bar{\Omega}$;

$\left(\beta_{2}\right)$ There are positive constants $\Lambda_{1}$ and $\Lambda_{2}$ for which

$$
\Lambda_{1}|\xi|^{2} \leq \frac{1}{2} \sum_{i, j=1}^{N} a^{i j}(x) \xi_{i} \xi_{j} \leq \Lambda_{2}|\xi|^{2}, \quad \forall x \in \bar{\Omega}, \xi \in \mathbf{R}^{N} .
$$

Put

$$
A(x, \xi):=\frac{1}{2} \sum_{i, j=1}^{N} a^{i, j}(x) \xi_{i} \xi_{j}, \quad \forall(x, \xi) \in \bar{\Omega} \times \mathbf{R}^{N},
$$

and consider the elliptic operator in divergent form

$$
L[u]=\sum_{i, j=1}^{N} \frac{\partial}{\partial x_{j}}\left(a^{i j}(x) \frac{\partial u}{\partial x_{i}}\right) .
$$

As observed by De Nápoli and Mariani in [7], the map $A$ satisfies assumptions $\left(\alpha_{1}\right)-$ $\left(\alpha_{5}\right)$. At this point, let $\Omega$ be a nonempty bounded open subset of the Euclidean space $\left(\mathbf{R}^{N},|\cdot|\right)$ with boundary of class $C^{1}$, and let $k: \bar{\Omega} \rightarrow \mathbf{R}$ be a positive continuous 
function. Further, let us fix $q \in] 2,2^{*}[$ and consider the function $h: \mathbf{R} \rightarrow \mathbf{R}$ defined by

$$
h(t):= \begin{cases}1+|t|^{q-1} & \text { if }|t| \leq r, \\ \frac{\left(1+r^{2}\right)\left(1+r^{q-1}\right)}{1+t^{2}} & \text { if }|t|>r\end{cases}
$$

where $r$ is a fixed constant such that

$$
r>\max \left\{\kappa, q^{\frac{1}{q-2}}\left(G_{1}+G_{2}\right)^{\frac{1}{q-2}}\right\} .
$$

Clearly $h(0) \neq 0$ and $h(t) \leq\left(1+|t|^{q-1}\right)$ for every $t \in \mathbf{R}$. Hence, condition (5) is satisfied for $a_{1}=a_{2}=1$. Moreover, also condition $\left(\mathrm{h}_{1}\right)$ is verified since $\lim _{|t| \rightarrow+\infty} h(t) /|t|=0$. Finally, owing to (11), it follows that

$$
G_{1}+G_{2}<\frac{r^{q-2}}{q}
$$

Therefore,

$$
\frac{H(r)}{r^{2}}=\frac{r^{q-2}}{q}+\frac{1}{r}>G_{1}+G_{2}
$$

where $H(r):=\int_{0}^{r} h(t) d t$, and condition $\left(\mathrm{h}_{2}\right)$ holds choosing $\delta=r$.

Consequently, owing to Theorem 3.5, for each parameter $\lambda$ belonging to the interval

$$
\left.\Lambda_{\delta}:=\frac{4\left(2^{N}-1\right) \Lambda_{2}}{\tau^{2} \min _{x \in \bar{\Omega}} k(x)}\right] \frac{r^{2}}{H(r)}, \frac{1}{G_{1}+G_{2}}[,
$$

the following elliptic Dirichlet problem

$$
\left(\widetilde{D}_{\lambda}^{h}\right) \quad \begin{cases}-L[u]=\lambda k(x) h(u) & \text { in } \Omega \\ u=0 & \text { on } \partial \Omega\end{cases}
$$

possesses at least three nontrivial weak solutions in $H_{0}^{1}(\Omega)$.

Remark 3.13. We observe that, to the contrary to the result of Kristály, Lisei and Varga studied [10, Theorem 2.1], in Theorem 3.5, as for instance Example 3.12 shows, we don't require the following behaviour at zero

$$
\lim _{t \rightarrow 0} \frac{f(t)}{|t|^{p-1}}=0
$$

that automatically implies $f(0)=0$.

Moreover, for completeness, we emphasize that our results can be investigated also for different classes of elliptic operators in divergence form looking at the existence of at least three nontrivial weak solutions. See, for instance, the recent and interesting paper of Colasuonno, Pucci, and Varga [5] for related topics.

Acknowledgements. The authors warmly thanks the anonymous referees for their useful and nice comments on the manuscript. The research was supported in part by the SRA grants P1-0292-0101 and J1-4144-0101. Moreover, the first author is supported by the GNAMPA Project 2012 titled: Esistenza e molteplicità di soluzioni per problemi differenziali non lineari. 


\section{References}

[1] Autuori, G., P. Pucci, and Cs. Varga: Existence theorems for quasilinear elliptic eigenvalue problems in unbounded domains. - Adv. Differential Equations 18, 2013, 1-48.

[2] Bonanno, G., and S. A. Marano: On the structure of the critical set of non-differentiable functions with a weak compactness condition. - Appl. Anal. 89, 2010, 1-10.

[3] Bonanno, G., and G. Molica Bisci: Three weak solutions for Dirichlet problems. - J. Math. Anal. Appl. 382, 2011, 1-8.

[4] BrÉzis, H., and L. Oswald: Remarks on sublinear elliptic equations. - Nonlinear Anal. 10, $1986,55-64$.

[5] Colasuonno, F., P. Pucci, and Cs. Varga: Multiple solutions for an eigenvalue problem involving $p$-Laplacian type operators. - Nonlinear Anal. 75, 2012, 4496-4512.

[6] D'Aguì, G., and G. Molica Bisci: Three non-zero solutions for elliptic Neumann problems. - Anal. Appl. (Singap.) 9:4, 2011, 383-394.

[7] De Nápoli, P., and M. C. Mariani: Mountain pass solutions to equations of $p$-Laplacian type. - Nonlinear Anal. 54, 2003, 1205-1219.

[8] Duc, D. M., and N. T. Vu: Nonuniformly elliptic equations of $p$-Laplacian type. - Nonlinear Anal. 61, 2005, 1483-1495.

[9] Fan, X. L., and C. X. Guan: Uniform convexity of Musielak-Orlicz-Sobolev spaces and applications. - Nonlinear Anal. 73, 2010, 163-175.

[10] Kristály, A., H. Lisei, and Cs. Varga: Multiple solutions for $p$-Laplacian type equations. - Nonlinear Anal. 68, 2008, 1375-1381.

[11] Kristály, A., V. RĂDUlescu, and Cs. VARGA: Variational principles in mathematical physics, geometry, and economics: Qualitative analysis of nonlinear equations and unilateral problems. - Encyclopedia Math. Appl. 136, Cambridge Univ. Press, Cambridge, 2010.

[12] Lin, S. S.: On the number of positive solutions for nonlinear elliptic equations when a parameter is large. - Nonlinear Anal. 16, 1991, 283-297.

[13] Molica Bisci, G.: Some remarks on a recent critical point result of nonsmooth analysis. Matematiche (Catania) 64, 2009, 97-112.

[14] Papageorgiou, N. S., E. M. Rocha, and V. Staicu: A multiplicity theorem for hemivariational inequalities with a $p$-Laplacian like differential operator. - Nonlinear Anal. 69, 2008, $1150-1163$.

[15] Perera, K., P. Pucci, and Cs. Varga: An existence result for a class of quasilinear elliptic eigenvalue problems in unbounded domains. - Preprint.

[16] Servadei, R.: Existence results for semilinear elliptic variational inequalities with changing sign nonlinearities. - NoDEA Nonlinear Differential Equations Appl. 13, 2006, 311-335.

[17] Talenti, G.: Best constants in Sobolev inequality. - Ann. Mat. Pura Appl. (4) 110, 1976, $353-372$.

[18] YAng, Z., D. Geng, and H. Yan: Three solutions for singular $p$-Laplacian type equations. Electron. J. Differential Equations 2008:61, 2008, 1-12.

Received 12 February 2013• Accepted 5 August 2013 